\title{
Development and Optimization of Design of an Acceleration Sensor Using Ferrofluid
}

\author{
Dong-Ying Ju* and Kazuaki Tabata ${ }^{1}$ \\ Department of Material Science and Engineering, Saitama Institute of Technology, \\ Fusaiji 1690, Fukaya, Saitama, Japan \\ ${ }^{1}$ Institute of Advanced Science, Saitama Institute of Technology, Japan
}

(Received August 15, 2008; accepted October 1, 2008)

Key words: magnetite nanoparticles, magnetic fluid, magnetic properties, acceleration sensor, optimization

A new electromagnetic-type acceleration sensor using ferrofluid was developed to improve the sensitivity and decrease energy consumption. To develop a small acceleration sensor, numerical simulation was carried out. On the basis of the simulation results, the parameters of the sensor were optimized, and a $10 \times 10 \times 10 \mathrm{~mm}^{3}$ acceleration sensor was fabricated in this project. The linear measurement scope of the sensor was also confirmed. It was also confirmed that the sensitivity of this sensor was better than those of previous sensors.

\section{Introduction}

The position of a high-speed train, an automobile and a manufacturing machine during movement and measurements of moving velocity and acceleration become convenient with the development of satellite communication. However, global positioning system (GPS) cannot be used in areas where the electric waves of an underground train, a tunnel, and an underwater tube exists, although GPS is a system that measures the positional coordinates of the ground using satellite. Generally, it is considered that the detection of the position, velocity, and acceleration of a moving machine is important because the safety of key equipment is secured in areas of disasters such as earthquakes. If the inertia navigation system is applied to the movement measurement of the human body, mechanics information on acceleration, velocity, amount of movement, rotation corner, posture, and inclination can be obtained.

Moreover, a small acceleration sensor can be used for medical treatment. A patient can train while voluntarily observing the indicator when it is possible to apply a position sensor and an acceleration sensor to all the systems that measure the movement of the human body, like sports measurement and rehabilitation training, and it can be used for rehabilitation; a new target can also be made in training. On the other hand, a small acceleration sensor can be used in a navigation system so that the position and movement of a moving machine and humans can be measured.

*Corresponding author: e-mail: dyju@sit.ac.jp 
Ferrofluids are composed of nanoscale ferromagnetic or ferrimagnetic particles suspended in a carrier fluid, usually an organic solvent, such as oil or water. The particles in a ferrofluid primarily consist of nanoparticles that are suspended by Brownian motion and generally will not settle under normal conditions. ${ }^{(1,2)}$ Permanent magnet sensors are well adapted in many systems, because they are contactless, robust, and relatively inexpensive. ${ }^{(3-9)}$ Recently, for the advancement of science and society, the acceleration sensor has been used in many industrial fields, such as in machines, airplanes, cars, and trains. Today, most acceleration sensors are fabricated by MEMS technology. ${ }^{(10-}$ 18) To develop an acceleration sensor with high sensitivity, high reliability, low cost and low energy consumption, a new type of acceleration sensor that uses magnetic fluid is proposed in this paper. In this research, we examine the effect of magnetic fluid use on the acceleration sensor. Parameters such as the shape and size of the magnet, coercive force, coil number, and position were optimized on the basis of simulation results. The performance of the sensor was confirmed by an actual test.

The design of a necessary sensor for an inertia navigation system was carried out, to achieve the miniaturization of an induction device with a sensor that uses the developed ferrofluid with magnetic nanoparticles of about $8 \mathrm{~nm}$ size. ${ }^{(19)}$ The main feature of the sensor that uses this ferrofluid is that the power consumption is extremely minimal, and lightening the entire device is possible. The accelerometer that uses ferrofluid was easy to miniaturize with an accuracy of a previous servo accelerometer, a small power consumption, and a calibration-free feature. It was confirmed that the rotation corner accelerometer can be realized by applying the actuator mechanism of this accelerometer. In this study, the research and development of a rotation corner accelerometer that uses ferrofluid and has low power consumption was carried out.

\section{Measurement Principle of Acceleration}

As principle of this sensor, we mainly use the relationship between force, current and magnetic field during ferrofluid movement. In a magnetic field, the force $F$ can be shown from the Law of Coulomb as

$$
\vec{F}=q_{\mathrm{m}} \times \vec{H},
$$

where $q_{\mathrm{m}}$ is the magnetic charge. The magnetic field $B$ is governed by

$$
\vec{B}=\mu_{0} \times \vec{H},
$$

where the initial permeability $\mu_{0}$ is constant. When the ferrofluid is not deformed (or slightly deformed), the force due to inertia force can be shown as

$$
m \vec{a}=\vec{F}=q_{\mathrm{m}} \times \mu_{0} \vec{B}
$$

therefore, the acceleration of an actuator in a magnetic field can be measured using this principle. In this study, a new type of acceleration sensor $\left(10 \times 10 \times 10 \mathrm{~mm}^{3}\right)$ has been 
fabricated as shown on Fig. 1. The principle diagram of the acceleration sensor is shown in Fig. 2. In the sensor structure, the balance relation of the forces on the actuator is shown as

$$
m \vec{a}=\vec{F}_{1}+\vec{F}_{2}+\vec{F}_{3}+\vec{F}_{4}
$$

When a current-carrying conductor is placed in a magnetic field $\vec{B}$, the force $\vec{F}$ that the conductor receives from the magnetic field is calculated using

$$
F=I B L
$$

where $I$ is the current in the coil and $L$ is the distance moved by the actuator. When the magnet is offset from the center caused by acceleration and yet maintains balance, then acceleration can be calculated using eq. (5).

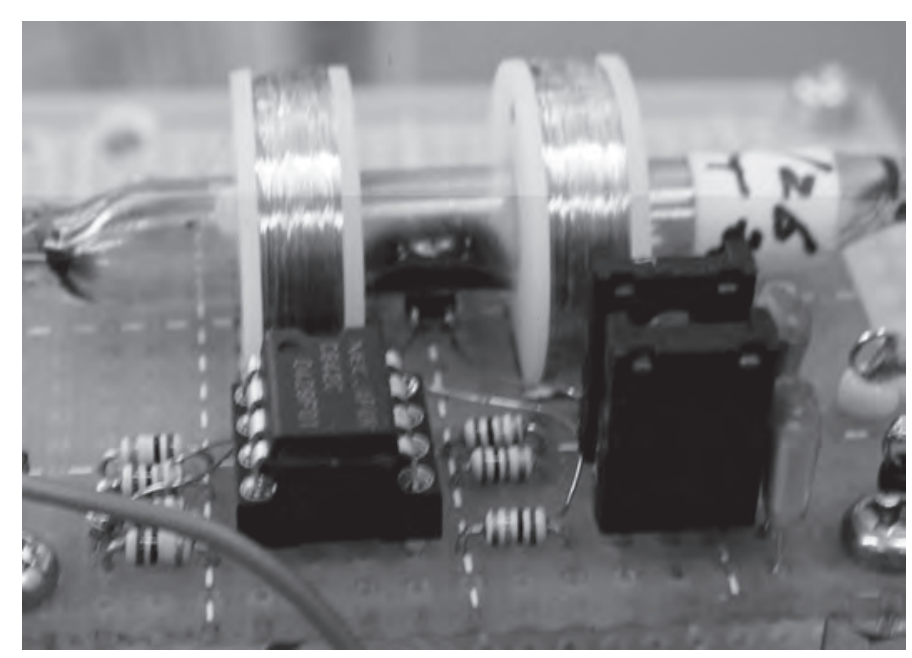

Fig. 1. Acceleration sensor.

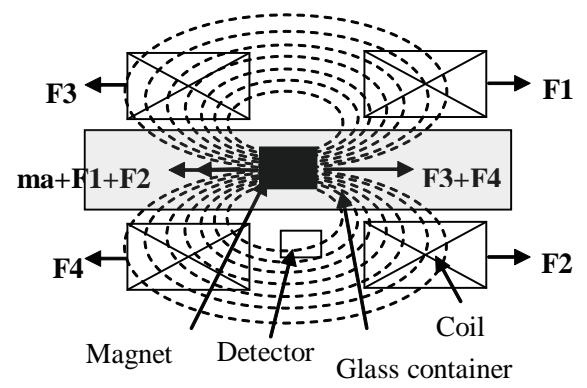

Fig. 2. Principle diagram of acceleration sensor. 
In this research, we proposed the mechanism of a small servo actuator that is common to the acceleration sensor and rotation corner acceleration sensor, and is used at high powers. Moreover, a drive circuit was designed. In addition, a good detector coil and a detector circuit were designed and compared of their $\mathrm{S} / \mathrm{N}$ values.

\section{Simulation and Optimizing Design}

Because the magnetic field of a magnet is very strong and the magnetic field of coils is much lower than $1 \%$, the influence of coils can be neglected.

In this research, we analyzed the magnetic field to establish the optimum conditions for determining the effects (3) of the amount of (1) ferrofluid and (2) coil rolling using ANSYS, and compared the analytical results. Figure 2 shows the structure and modeling of the acceleration sensor that is used to analyze magnetic field. The material characteristics are shown in Table 1.

Figures 3(a) and 3(b) show the magnetic fields without and with a fully magnetic fluid, respectively. The magnetic field near the ends of the magnet is so strong that a large acting force is exerted on both sides. If a suitable magnetic fluid is added, a capshaped figure appears on both ends. Therefore, magnetic fluid can improve lubrication and sensitivity. A hall element detector can detect magnetic flux density itself and output voltage. Simulation results of distance offset from the center as well as of magnetic

Table 1

Acceleration sensor calculation parameters.

\begin{tabular}{llll}
\hline Coils & 3000 & Magnet (permeability) & $1 \mathrm{~N} \cdot \mathrm{A}^{-2}$ \\
\hline Max. I & $0.3 \mathrm{~mA}$ & Air (permeability) & $1 \mathrm{~N} \cdot \mathrm{A}^{-2}$ \\
\hline Magnet mass & $0.1 \mathrm{~g}$ & Coils (permeability) & $1 \mathrm{~N} \cdot \mathrm{A}^{-2}$ \\
\hline Magnetic fluid & B-H curve & & \\
\hline
\end{tabular}

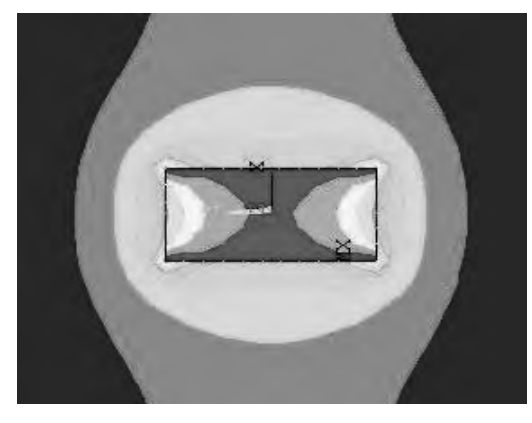

(a)

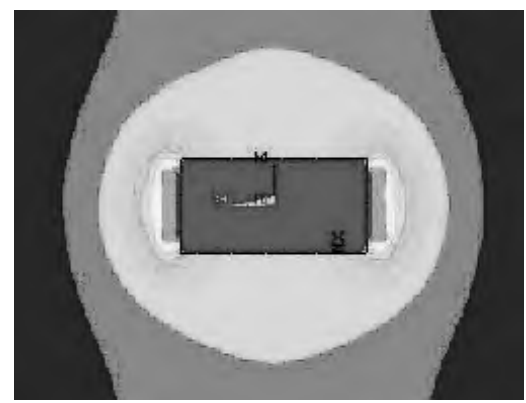

(b)

Fig. 3. Magnetic field (a) without and (b) with fully magnetic fluid. 
flux density, magnetic flux density and acceleration relations are shown in Figs. 4 and 5, respectively.

Figure 6 shows the relationship between output hall voltage and magnetic flux density. The effect of magnet position on magnetic induction is shown in Fig. 7. From these results, we can determine that the sensor has a good linear relationship between output hall voltage and magnetic field. On the other hand, the relationship between the distance moved from the center and the detected magnetic induction, and the effect of magnet position on magnetic field are shown in Figs. 7 and 8. In the magnetic field, the movement behavior of the magnet actuator is also linear, so that the acceleration sensor can give a linear relationship between the distance moved from the center and the detected magnetic induction.

To examine the change in the magnetic induction induced by the movement range and the movement of the oscillator $4 \mathrm{~mm}$ in length. Tamotsu magnetisms $3 \times 10^{5} \mathrm{~A} / \mathrm{m}$ were input and magnetic field was analyzed. Figures 4 and 5 show the results. It has been understood that acceleration behavior is a line within the movement range $(-1,1)$

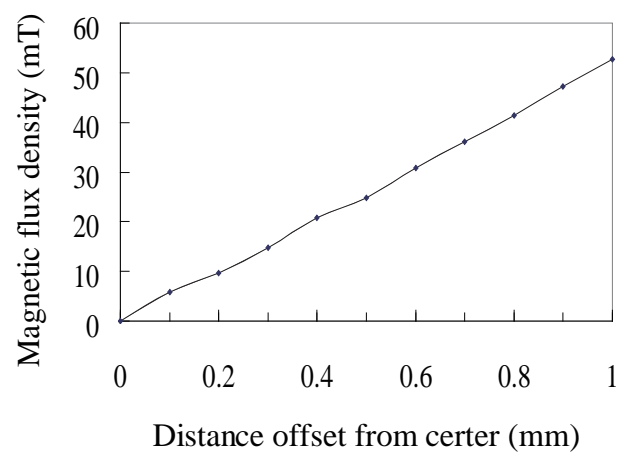

Fig. 4. Relationship between distance offset from center and magnetic flux density.

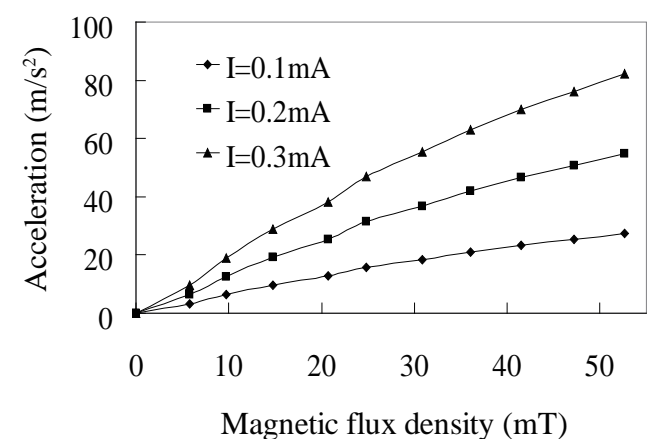

Fig. 5. Relationship between magnetic flux density and acceleration. 


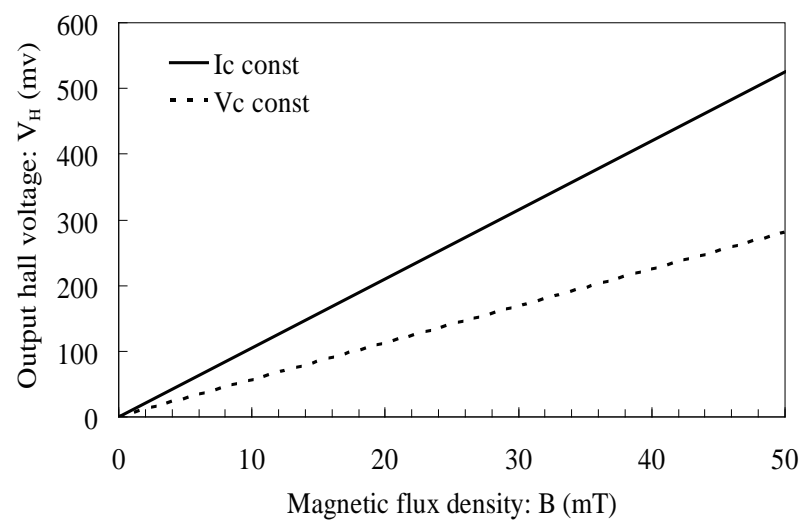

Fig. 6. Relations between output hall voltage and magnetic flux density.
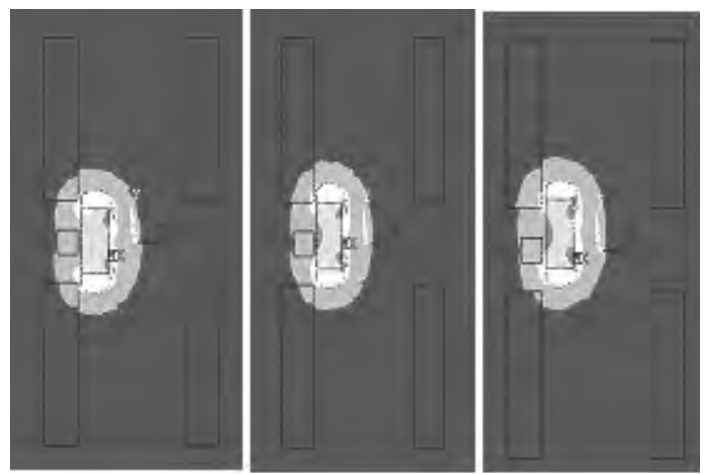

Fig. 7. Effect of magnet position on magnetic induction.

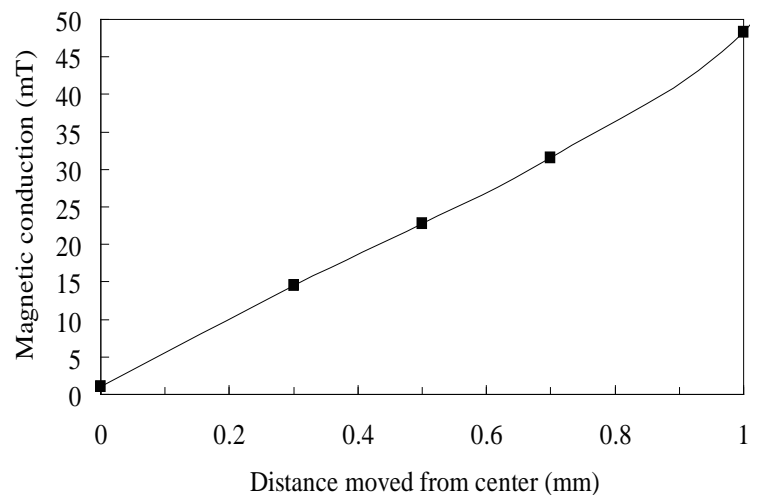

Fig. 8. Relations between distance moved from center and detected magnetic induction. 
$\mathrm{mm}$ of the oscillator. Acceleration can be calculated from the magnetic induction that detects the hall element. Moreover, the magnetic induction range is $(-350,350) \mathrm{mT}$. The time base range of the hall on the market element is $(-50,50) \mathrm{mT}$, as shown in Fig. 6, and the magnetism of the magnet has been adjusted for that to $5 \times 10^{4} \mathrm{~A} / \mathrm{m}$. Figure 7 shows the result of the analysis. It is understood that the detection range of magnetic induction is $(-50,50) \mathrm{mT}$ from the result obtained within a small range $(-1,1) \mathrm{mm}$ of the oscillator.

Figure 8 shows an analytical result of the oscillator receiving power. Acceleration can be determined using eq. (4). Moreover, the time base range of the acceleration sensor is shown in Fig. 8. Because the time base range of the acceleration sensor also changes, when the current in the driving coil increases slightly, it is understood that the time base range of the sensor for the ferrofluid acceleration is broad.

Figure 9 shows the time base range of acceleration. It is understood that the time base range of the sensor for ferrofluid acceleration changes marked with the current change of the driving coil.

From results of the above analyses, it seems that a sensor smaller than previous acceleration sensors (about $10 \times 10 \mathrm{~mm}^{2}$ ) can be fabricated. Moreover, the size, number and volume of sensors, the length and magnetism of the magnet, the distance moved by the oscillator, and the necessary conditions for setting the current in the driving coil, and other factors have been understood.

When there is a ferrofluid change in a magnet surrounding magnetic induction and when there is no ferrofluid, lines of magnetic force concentrate at the edge, and the change in magnetic induction grows. A slight movement of the oscillator can be detected. It has been understood from the above discussion that the sensitivity of an acceleration sensor increases with the addition of a ferrofluid.

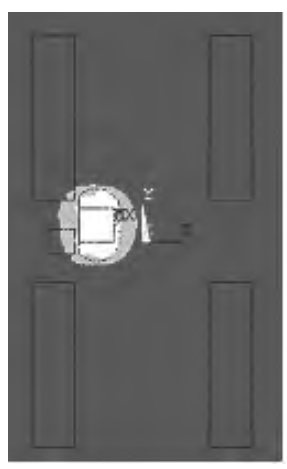

(a) Center

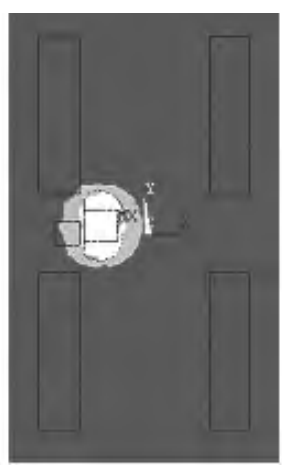

(b) $0.5 \mathrm{~mm}$ off center

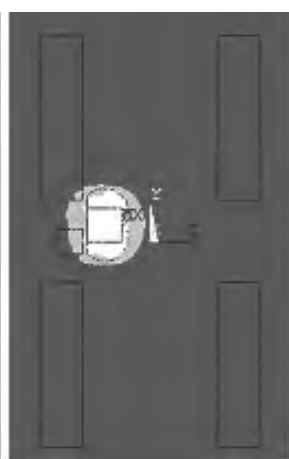

(c) $1 \mathrm{~mm}$ off center

Fig. 9. Effect of magnet position on magnetic induction. Magnet length, $2 \mathrm{~mm}$; saturation magnetization value, $3 \times 10^{5} \mathrm{~A} / \mathrm{m}$. 


\section{Conclusions}

In this research, we developed a new acceleration sensor that uses ferrofluid. Moreover, we determined the parameters of the sensor by analyzing magnetic field using the finite element method. In the study, following finding was obtained. Magnetic fluid can improve lubrication and sensitivity.

The relationships between the linear measurement scope and the material properties were confirmed on the basis of simulation results. A $10 \times 10 \times 10 \mathrm{~mm}^{3}$ acceleration sensor that is $1 / 2$ the size of original design can be fabricated.

Optimizing coil radius and coil length, as well as the total number of turns, is necessary in developing the sensor. The optimum conditions for improving the resolution of this sensor were obtained from the simulation result of this research.

\section{Acknowledgements}

The support provided by the High Technology Research Center and Open research center in Saitama Institute of Technology for this work is gratefully acknowledged.

\section{References}

1 J. P. Yonnet, A. Foggia and S. Adenot: 15th Int. Workshop on Rare Earth Magnets and Their Applications, Dresden, September (1998) p. 847.

2 S. Adenot, J. P. Yonnet and A. Foggia: 14th Int.Workshop on Rare Earth Magnets and Their Application, San Paulo, September (1996) p. 624.

3 T. Kaiath: Linear Systems (Prentice-Hall, Inc. Englewood Cliffs, N.J., 1980).

4 Z. Deng, T. J. Carlson, J. P. Duncan and M. C. Richmond: Sensors 7 (2007) 3399.

5 D. S. Park, D. J. Yun, M.W. Cho and B. C. Shin: Sensors 7 (2007) 697.

6 Y. H. Cho: Journal of the Korean Society for Precision Engineering 17 (2000) 52.

7 L. M. Roylance and J. B. Angell: IEEE Trans. Electron Devices 26 (1979) 1887.

8 M. A. Schmidt and R. T. Howe: Engineering Science Proceedings 8 (1987) 1911.

9 E. Rudilf, A. Jornod, J. Bergqvist and H. Leuthold: Sens. Actuators, A 21 (1990) 297.

10 Y. Matsumoto and M. Esashi: Sens. Actuators, A 39 (1993) 209.

11 W. Kuehnel and S. Sherman: Sens. Actuators, A 45 (1999) 7.

12 L. Parameswaran, C. H. Hsu and M. A. Schmidt: Proceedings of Transducers (1997) 625.

13 B. P. van Drieehuizen, N. I. Maluf, I. E. Opris and G. T. A. Kovacs: Proceedings of Transducers (1997) 1229.

14 E. Belloy, A. Sayah and M. A. M. Gijs: Journal of Microelectrochemical Systems 11 (2002) 85.

15 E. Belloy, S. Thurre, E. Walckiers, A. Sayah and M. A. M. Gijs: Sens. Actuators, A 84 (2000) 330.

16 E. Belloy, A. Sayah and M. A. M. Gijs: Sens. Actuators, A 86 (2000) 231.

17 P. Slikkerveer, P. Bouten and F. de Haas: Sens. Actuators, A 85 (2000) 296.

18 B. Sebastian: Journal of Microelectromechanical Systems 9 (2000) 551.

19 P. Bian and D.Y. Ju: 12th Int. Symp. on Interdisciplinary Electromagnetic, Mechanics \& Biomedical Problems, Sept. 12-14, Bad Gastein, Austria (2005) p. 12. 